Bot. Mag. Tokyo 84: 293 298, 1971

\title{
Four Karyotypes of Rumex acetosa L.
}

\author{
Masahide Kurita and Y Ûzô Kuroki
}

Biological Institute, Ehime University, Matsuyama, 790

\begin{abstract}
A study was carried out on karyotypes of four clones of Rumex acetosa L.
Their karyotypes were formulated as follows:

$$
\begin{aligned}
2 \mathrm{n}=15 & =\mathrm{X}^{m}+\mathrm{X}_{1}{ }^{m}+\mathrm{Y}_{2}{ }^{s m}+7 \mathrm{~A}^{s t}+2^{t} \mathrm{~A}^{s t}+\mathrm{A}^{s t>}+\mathrm{a}^{s m}+\mathrm{a}^{s t} \\
\prime \prime & =\mathrm{X}^{m}+\mathrm{Y}_{1} m+\mathrm{Y}_{2}^{s m}+7 \mathrm{~A}^{s t}+\mathrm{A}^{s m}+2^{t} \mathrm{~A}^{s t}+\mathrm{A}^{s t}>+\mathrm{a}^{m} \\
2 \mathrm{n}=14 & =2 \mathrm{X}^{m}+\mathrm{A}^{m}+6 \mathrm{~A}^{s t}+2^{t} \mathrm{~A}^{s t}+2 \mathrm{a}^{s m}+\mathrm{ma}^{s m} \\
\prime \prime & =2 \mathrm{X}^{m}+7 \mathrm{~A}^{s t}+2 \mathrm{~A}^{s m}+{ }^{t} \mathrm{~A}^{s t}+{ }^{t} \mathrm{a}^{s m}+\mathrm{a}^{s m}
\end{aligned}
$$

(st $>$ and ma representing a subterminal type approximating to terminal one and an extremely small chromosome respectively)

These karyotypes seem to have resulted through intra- and inter-chromosomal translocation, and the first karyotype is assumed to be one of the most advanced karyotypes in diploid clone.
\end{abstract}

There are recent papers (Kuroki and Kurita, 1969a, '70a-b) dealing with over 15 different karyotypes of Rumex acetosa L. Further study has shown us four peculiar karyotypes each of which is plainly distinguishable from any one described before. In this paper, there will be given full details of those four karyotypes.

\section{Materials and Methods}

The materials used are two male clones (No. 300 collected from Inagi-cho, Tokyo Metrop., and No. 321 from Tottori City, Tottori Pref.) and two female clones (No. 363 collected from Kaimon-cho, Kagoshima Pref., and No. 327 from Nakanojo-cho, Gunma Pref.). Cytological preparations were made from root tips following the same procedure as employed in our early study (Kuroki and Kurita, 1969a).

All the symbols of chromosomes, including two new symbols which will be presented later, are also used in the same ways as interpreted formerly (Kuroki and Kurita, 1969a). The autosomes of $R$. acetosa were classified, by the authors (1969a), into two groups: A and a. In this paper, these two groups are distinguished from each other only by a difference in length of long arm which is, as mentioned already (Kuroki and Kurita, 1970b), the most important condition for distinguishing: the group-A chromosome whose long arm being large and the group-a chromosome whose long arm small.

\section{Results}

Clone No. 300 (Table 1 and Figs. 1, 5): This clone has 12 autosomes besides the sex chromosomes: $\mathrm{X}, \mathrm{Y}_{1}$ and $\mathrm{Y}_{2}$. These sex chromosomes each show an ordinary type, 
namely the $\mathrm{X}$ and $\mathrm{Y}_{1}$ are of median type and the $\mathrm{Y}_{2}$ is of submedian type. Those autosomes are classified into four main groups as follows: 1) Seven group-A chromosomes of subterminal type (Table 1 and Fig. 1:1-4, 8-10). 2) Two group-A chromosomes of subterminal type furnished with satellites on their short arms (ibid. 5, 6). 3) One group-A chromosome whose short arm is minute (ibid. 7). It is of exceedingly subterminal type approximating to terminal one. 4) Two group-a chromosomes. One is of submedian type (ibid. 11) and the other, having a minute arm, is of exceedingly subterminal type (ibid. 12). The former chromosome is occasionally found to be slightly smaller in length of long arm than the latter chromosome. This minute arm is quite a bit larger than that of the chromosome in the third group.

Table 1. Measurement of chromosomes in Rumex acetosa ( $\hat{\delta}$, clone No. 300). $\mathrm{t}$ : satellite; ma: minute arm; *: arm-ratio.

\begin{tabular}{c|c|c|c}
\hline \hline Chrom. & Length of arm $(\mu)$ & Total & Index* \\
\hline $\mathrm{X}$ & $4.4+4.1$ & 8.5 & 0.93 \\
$\mathrm{Y}_{1}$ & $3.8+3.4$ & 7.2 & 0.89 \\
$\mathrm{Y}_{2}$ & $3.9+2.2$ & 6.1 & 0.56 \\
1 & $4.3+1.0$ & 5.3 & 0.23 \\
2 & $4.2+1.0$ & 5.2 & 0.24 \\
3 & $4.2+0.7$ & 4.9 & 0.17 \\
4 & $3.9+0.9$ & 4.8 & 0.23 \\
5 & $4.1+0.7 \ldots \mathrm{t}$ & 4.8 & 0.17 \\
6 & $3.8+0.8 \ldots \mathrm{t}$ & 4.6 & 0.21 \\
7 & $4.8+\mathrm{ma}$ & 4.8 & 0.21 \\
8 & $3.9+0.8$ & 4.7 & 0.29 \\
9 & $3.4+1.0$ & 4.4 & 0.24 \\
10 & $3.4+0.8$ & 4.2 & 0.42 \\
11 & $2.4+1.0$ & 3.4 & 0.11 \\
12 & $2.7+0.3$ & 3.0 & \\
\hline
\end{tabular}

Then, using the symbol st> standing for a near-terminal type, the karyotype of this clone is formulated as follows: $2 \mathrm{n}=15=\mathrm{X}^{m}+\mathrm{Y}_{1}{ }^{m}+\mathrm{Y}_{2}{ }^{s m}+7 \mathrm{~A}^{s t}+2^{t} \mathrm{~A}^{s t}+\mathrm{A}^{s t}>+\mathrm{a}^{s m}+$ $a^{s t}$.

Clone No. 321 (Figs. 2, 6): This clone has also 12 autosomes besides the sex chromosomes: $\mathrm{X}, \mathrm{Y}_{1}$ and $\mathrm{Y}_{2}$. Each of the sex chromosomes is of an ordinary type. The 12 autosomes fall into five main groups. 1) Seven group-A chromosomes of subterminal type (Fig. 2: 1, 2, 4, 7-10). Out of their long arms, the smallest one is about $3.2 \mu$ in length. 2) One group-A chromosome of submedian type having the index of about 0.59 (ibid. 3). 3) Two group-A chromosomes of subterminal type (ibid. 5, 6) showing satellites on their short arms. One satellite (ibid. 5) is larger than the other (ibid. 6). 4) One group-A chromosome (ibid. 11) of exceedingly subterminal type near to terminal one. Its long arm is about $2.7 \mu$ in length and its short arm is as minute as that of the autosome of near-terminal type (Fig. 1, 7) in the just foregoing clone. 5) One group-a chromosome (ibid. 12) of median type, averaging $1.9 \mu+1.7 \mu$.

The karyotype formula of this clone is as follows: $2 \mathrm{n}=15=\mathrm{X}^{m}+\mathrm{Y}_{\mathbf{1}}{ }^{m}+\mathrm{Y}_{\mathbf{2}}{ }^{\mathrm{m}}+$ $7 \mathrm{~A}^{s t}+\mathrm{A}^{s m}+2^{t} \mathrm{~A}^{s t}+\mathrm{A}^{s t}>+\mathrm{a}^{m}$. 

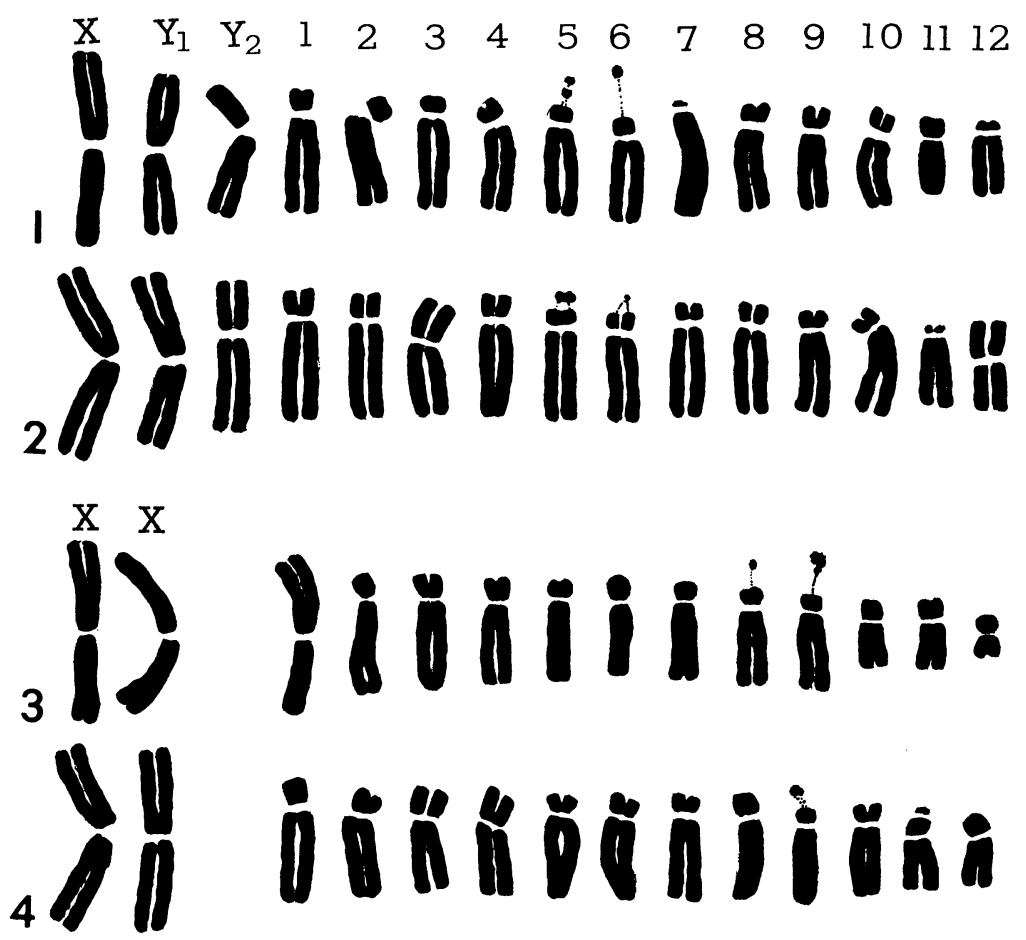

Figs. 1-4. Alignement of metaphase chromosomes. Fig. 1: clone No. 300; Fig. 2: No. 321; Fig. 3: No. 363; Fig. 4: No. 327. $\times 2270$.

Table 2. Measurement of chromosomes in Rumex acetosa ( , clone No. 363). t: satellite; *: arm-ratio.

\begin{tabular}{c|c|c|c}
\hline \hline Chrom. & Length of arm $(\mu)$ & Total & Index* \\
\hline X & $4.1+4.0$ & 8.1 & 0.98 \\
X & $4.2+3.9$ & 8.1 & 0.93 \\
1 & $3.8+3.4$ & 7.2 & 0.89 \\
2 & $4.3+1.1$ & 5.4 & 0.26 \\
3 & $4.1+1.0$ & 5.1 & 0.24 \\
4 & $3.6+1.0$ & 4.6 & 0.28 \\
5 & $3.7+0.8$ & 4.5 & 0.22 \\
6 & $3.4+1.1$ & 4.5 & 0.32 \\
7 & $3.4+0.8$ & 4.2 & 0.24 \\
8 & $3.4+0.7 \ldots \mathrm{t}$ & 4.1 & 0.21 \\
9 & $3.4+0.7 \ldots \mathrm{t}$ & 4.1 & 0.21 \\
10 & $2.1+1.0$ & 3.1 & 0.48 \\
11 & $2.0+1.0$ & 3.0 & 0.50 \\
12 & $1.1+0.8$ & 1.9 & 0.73 \\
\hline
\end{tabular}

Clone No. 363 (Table 2 and Figs. 3, 7): This clone is found to have two X-chromosomes and 12 autosomes. Each X-chromosome shows an ordinary type. The 12 autosomes are classified into five main groups. 1) One group-A chromosome of median type (Fig. 3, 1). This chromosome is quite a bit smaller than the $\mathrm{X}$ and is similar in shape and size to the $Y_{1}$ in male. However, it is not found to be more condensed than 
any other chromosome at mid-prophase. 2) Six group-A chromosomes of subterminal type (ibid. 2-7). 3) Two group-A chromosomes of subterminal type bearing satellites (ibid. 8, 9). The satellites differ slightly in size from each other. 4) Two group-a chromosomes of submedian type (ibid. 10, 11). 5) Extremely small chromosome of submedian type (ibid. 12). This is always one in number per nucleus and is found at every metaphase.

The karyotype, using the symbol ma representing a chromosome which is as small as the chromosome in the fifth group, is formulated as follows: $2 n=14=2 \mathrm{X}^{m}+\mathrm{A}^{m}$ $+6 \mathrm{~A}^{s t}+2^{t} \mathrm{~A}^{s t}+2 \mathrm{a}^{s m}+m \mathrm{a}^{s m}$.

Clone No. 327 (Figs. 4, 8): This clone has two X-chromosomes of ordinary type and 12 autosomes. These autosomes are classified into four main groups. 1) Seven group-A chromosomes of subterminal type (Fig. 4: 1, 2, 5-8, 10). 2) Two group-A chromosomes of submedian type (ibid. 3,4) showing the index of about 0.47. 3) One group-A chromosome of subterminal type with satellite (ibid. 9). 4) Two group-a chromosomes of submedian type (ibid. 11, 12). One of them bears a satellite on its short arm, having the index of 0.37 (ibid. 11), and the other has no satellite, showing the index of 0.40 (ibid. 12).

The karyotype of this clone is represented as follows: $2 \mathrm{n}=14=2 \mathrm{X}^{m}+7 \mathrm{~A}^{s t}+$ $2 \mathrm{~A}^{s m}+{ }^{t} \mathrm{~A}^{s t}+{ }^{t} \mathrm{a}^{s m}+\mathrm{a}^{s m}$.

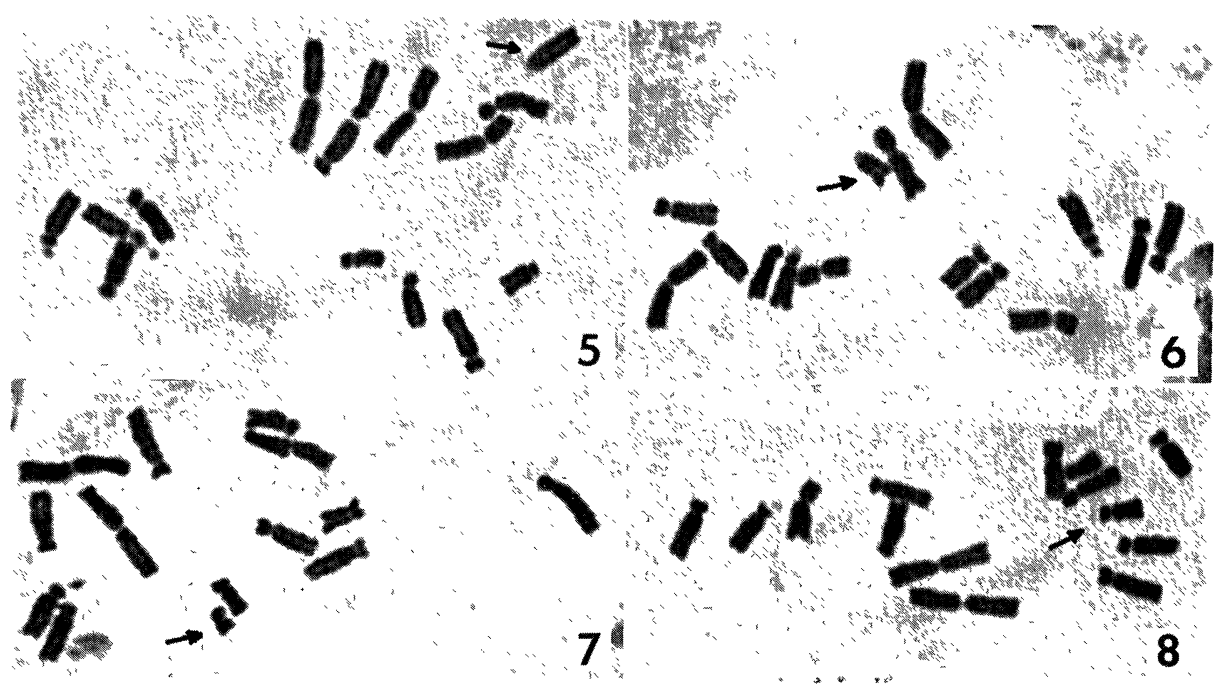

Figs. 5-8. Microphotographs of metaphase chromosomes. Fig. 5: clone No. 300; Fig. 6: No. 321; Fig. 7: No. 363; Fig. 8: No. 327. Arrow indicating $A^{\text {st }}>$-chromosome in Figs. 5 and $6, \mathrm{ma}^{\mathrm{sm}}$ in Fig. 7 , and ${ }^{\mathrm{t}} \mathrm{a}^{\mathrm{sm}}$ in Fig. $8 . \times 1300$.

\section{Discussion}

By Yamamoto (1933, '37, '38), certain karyotypes were explained to have arisen through a translocation. The four karyotypes in this paper can also be explained by 
the similar way, though meiosis is not yet analyzed.

In clone No. 300, there was found one group-A chromosome (Fig. 1, 7) which, having a minute arm, is extremely asymmetrical. It may be thought that a part called a minute arm consists really of centromeric chromomeres and that the chromosome is exactly of terminal type. By the authors, chromomere at centromere is, however, not yet confirmed in any chromosome of Rumex acetosa. Therefore, the part in question is tentatively described as a minute arm in this paper. It may be considered that the above group-A chromosome with minute arm has arisen through the transfer of the great part of short arm to long arm within an ordinary group-A chromosome. A group-a chromosome of median type has been observed to have the arm of which the distal region is heterochromatic, while a group-a chromosome of submedian type has not been observed to have such a heterochromatic region (Kuroki and Kurita 1969b, 70a). The subterminal type group-a chromosome (ibid. 12) found in clone No. 300 has also no heterochromatic region both in long and short arm. As shown in Table 1, the long arm of the subterminal type group-a chromosome (Table 1, 12) is slightly larger than that of the submedian type group-a chromosome (ibid. 11). This may indicate that the former chromosome has been brought about by the transfer of distal region of short arm to long arm within the homologue for the latter chromosome.

The extremely asymmetrical group-A chromosome (Fig. 2, 11), which has no heterochromatic region, in clone No. 321 must also have resulted from such transfer as mentioned just before. This chromosome is similar in length of long arm to the smallest group-A chromosome (ibid. 10) rather than the median type group-a chromosome (ibid. 12), though it is similar in entire length to the group-a chromosome. Then, at present it is not included in the group a, but in the group A.

It may be considered that a very small chromosome of submedian type (Fig. 3, 12) found in clone No. 363 is B-chromosome which had fully been studied by Haga (1961). The very small chromosome, as noted already, occurs at every metaphase and its number is not variable with cell, against the B-chromosome noted by Haga. Furthermore, somatic autosome complement which requires 12 members is first completed after an addition of the very small chromosome to it. Then, this small chromosome is not an accessory chromosome, but one of regular members. The third largest chromosome in Fig. 3 which is given the number one, as noted already, is somewhat smaller than the $\mathrm{X}$-chromosome, and also is not found to be more condensed than any other chromosome at mid-prophase, though it is similar in morphology to $\mathrm{Y}_{1^{-}}$ chromosome. The third largest chromosome (Fig. 3, 1) is not an X-chromosome, but an autosome, and also it is not $\mathrm{Y}_{\mathbf{1}}$-chromosome. The two different chromosomes (Fig. $3: 1,12)$ mentioned above may have arisen through a simple reciprocal translocation between two group-A chromosomes of subterminal type, namely through the transfer of a great part of long arm in one group-A chromosome to the short arm of the other group-A chromosome.

The clone No. 327 has two satellites, three or more satellites being never observed. A small chromosome with satellite was observed by Watanabe (1958). The satellite 
of group-a chromosome in this paper, on the one hand, may have been transferred from a short arm of one satellited group-A chromosome of subterminal type. On the other hand, the group-a chromosome with satellite may have arisen through missing the outer region of long arm of one satellited group-A chromosome, the region being transferred to the long arm of one submedian group-a chromosome.

Among the autosome complements of clone No. 300 (Fig. 1), only one chromosome is of submedian type, other two are of exceedingly subterminal type and of the similar type approximating to terminal one, and the rest are of moderately subterminal type. At present, it is probably safe to say that this karyotype is one of the most asymmetrical karyotypes with strict regard for the type I and II reported by Yamamoto (1933). Assuming that an asymmetrical karyotype is more advanced than a symmetrical one (Stebbins, 1950), the karyotype of clone No. 300 may be said to be one of the most advanced karyotypes.

\section{References}

HAGA, T. 1961. Intra-individual variation in number and linear patterning of the chromosome, I. Proc. Jap. Acad. 37: 627-632.

Kuroki, Y. And M. Kurita. 1969a. Karyotypes of Rumex acetosa L. Mem. Ehime Univ., Sci., Ser. B, 6: 41-49.

$\begin{array}{r}\text { 1969b. Certain autosomes in Rumex acetosa L. ibid. 6: 51-56. } \\ \hline \text { AND } 1970 \mathrm{a} \text {. New karyotype of Rumex acetosa L. Bot. Mag. Tokyo 83: } \\ \text { 95-98. } \\ \hline \text { AND 1970b. Further note on the karyotypes of Rumex acetosa L. Mem. }\end{array}$ Ehime Univ., Sci., Ser. B, 6: 209-214.

Stebbins, G. L. 1950. Variation and evolution in plants. Columbia Univ. Press, New York. Watanabe, H. 1958. Exhibition at the 30th annual meeting of the genetic society of Japan. Yамамото, Y. 1933. Karyotypes in Rumex acetosa L. and their geographical distribution. Jap. Jour. Genet. 8: 264-274.

1937. On the offspring of simple translocation heterozygotic plants in Rumex acetosa L. Jap. Jour. Bot. 13: 53-54.

1938. Karyogenetische Untersuchungen bei der Gattung Rumex. Mem. Coll. Agric. Kyoto Imp. Univ. 43:1-59. 